\title{
Telmisartan improves vascular remodeling through ameliorating prooxidant and profibrotic mechanisms in hypertension via the involvement of transforming growth factor- $\beta 1$
}

\author{
PEIJIN SHANG ${ }^{1,2^{*}}$, TIANLONG LIU ${ }^{1 *}$, WENXING LIU ${ }^{1 *}$, YUWEN LI $^{1,3}$, FANG DOU $^{1}$, YIKAI ZHANG ${ }^{4}$, \\ LIJUAN SUN ${ }^{5}$, TIEJUN ZHANG ${ }^{6}$, ZHIHUI ZHU ${ }^{1}$, FEI MU ${ }^{1}$, YI DING ${ }^{1}$ and AIDONG WEN ${ }^{1}$ \\ ${ }^{1}$ Department of Pharmacy, Xijing Hospital, Fourth Military Medical University, Xi'an, Shaanxi 710032; \\ ${ }^{2}$ Department of Pharmacy, Chinese PLA 517 Hospital, Xinzhou, Shanxi 034000; ${ }^{3}$ Department of Pharmacy, \\ The First Affiliated Hospital of Soochow University, Suzhou, Jiangsu 215006; ${ }^{4}$ Department of Pharmacy, \\ Chinese PLA Shenyang General Hospital, Shenyang, Liaoning 110016; 5 Department of Ophthalmology, \\ Eye Institute of Chinese PLA, Xijing Hospital, Fourth Military Medical University, Xi'an, Shaanxi 710032; \\ ${ }^{6}$ Department of Neurosurgery, The First Affiliated Hospital of Soochow University, Suzhou, Jiangsu 215006, P.R. China
}

Received October 18, 2016; Accepted June 13, 2017

DOI: $10.3892 / \mathrm{mmr} .2017 .7177$

\begin{abstract}
Vascular remodeling is a common complication and pathological basis of hypertension. Transforming growth factor- $\beta 1$ (TGF- $\beta 1$ )/small mothers against decapentaplegic 3 $(\mathrm{Smad} 3)$ is considered a potential therapeutic target for vascular remodeling in hypertension. The present study aimed to demonstrate the antifibrotic effects of telmisartan and examined the potential mechanisms associated with its prevention of vascular remodeling. Spontaneously hypertensive rats (SHRs) were treated with telmisartan (20 mg/kg), and vascular contractility, reactivity and oxidative stress were preliminarily evaluated. Vascular pathological alterations and collagen deposition were assessed using hematoxylin and eosin, and Masson staining, respectively. The profibrotic factors, TGF- $\beta 1$ and Smad3 were evaluated using immunofluorescence and immunohistochemistry. The protein levels of TGF- $\beta 1 / \mathrm{Smad} 3$, phosphorylated ( $\mathrm{p}$-)Smad3, collagen-1 and $\alpha$-smooth muscle actin in the aorta were assessed using western blot analysis. It was found that telmisartan attenuated chronic vasoconstriction and oxidative stress in the SHRs, and improved vascular reactivity. Telmisartan also restored vascular pathological alterations and decreased collagen deposition. In the vascular
\end{abstract}

Correspondence to: Dr Yi Ding or Dr Aidong Wen, Department of Pharmacy, Xijing Hospital, Fourth Military Medical University, 15 Changle West Street, Xi'an, Shaanxi 710032, P.R. China

E-mail: dingyi.007@163.com

E-mail: adwen-2004@hotmail.com

${ }^{*}$ Contributed equally

Key words: vascular remodeling, antioxidant effect, angiotensin II, transforming growth factor- $\beta 1$, collagen deposition wall of the SHRs, telmisartan effectively decreased the protein levels of TGF- $\beta 1 / \mathrm{Smad} 3$ and $\mathrm{p}-\mathrm{Smad} 3$. Taken together, these findings indicated that telmisartan, with its antioxidant effect, prevented vascular remodeling in hypertension through preventing the TGF- $\beta 1 / \mathrm{Smad} 3$ signaling pathway.

\section{Introduction}

Vascular remodeling is a common complication and the pathological basis of hypertension and other cardiovascular diseases (1). It is typically characterized by rearrangement of vascular tissue, higher cross-sectional area, narrowing vascular lumen and increased collagen deposition (2), which initially may be protective and adaptive changes (3). However, normal artery structure is necessary for functional requirements. Long-term vascular remodeling in hypertension inevitably deteriorates vascular resistance, decreases lumen narrowing and vascular distensibility, depletes compensative capacity of the vasculature, and induces cardiovascular events (4). It has been shown that angiotensin II (Ang II) and reactive oxygen species (ROS) induce transforming growth factor $\beta 1$ (TGF- $\beta 1$ ) and are important in vascular disorders (5-7). The vascular walls are vulnerable to chronic vasoconstriction and oxidative damage. Therefore, agents antagonizing Ang II receptor 1 (AT1) or in combination with antioxidant effects may decrease the activation of TGF- $\beta 1$ and have a potential therapeutic effect on vascular remodeling in hypertension.

TGF- $\beta 1$ is an important profibrotic factor in the vascular remodeling of hypertension. It phosphorylates membrane receptors and cytoplasm transducers, predominantly of the small mothers against decapentaplegic (Smad) family, and regulates gene expression (8). Smad3 is important in exerting TGF- $\beta 1$-induced fibrotic processes (9). Typically, the TGF- $\beta 1 / \mathrm{Smad} 3$ pathway regulates extracellular matrix (ECM) accumulation and collagen deposition, in addition to vascular cell activation, differentiation and migration $(10,11)$. 
The neutralization of TGF- $\beta 1$ attenuates hypertension and improves vascular structure $(7,12,13)$. As reported previously, TGF- $\beta 1$ is closely associated with the renin-angiotensin system (RAS). Ang II can induce the activation and release of TGF- $\beta 1$ in a time- and dose-dependent manner (14). In this way, Ang II promotes vascular remodeling directly. By contrast, Ang II triggers the production of ROS $(15,16)$, which induces the activation of TGF- $\beta 1$ and is closely associated with vascular pathologies. AT1 inhibitor is reported to effectively prevent vascular profibrotic mechanism involvement of matrix metalloproteinases and TGF- $\beta 1$, and reduces vascular remodeling in hypertension (17). It is likely that telmisartan, as a targeted Ang II receptor inhibitor, not only effectively controls blood pressure, but also ameliorates vascular remodeling and fibrosis in hypertension.

The present study aimed to determine whether telmisartan exerts vasoprotective effects and antagonizes vascular remodeling in hypertensive animals, and to examine the potential mechanisms involved. Experiments were performed to assess vascular remodeling and fibrotic factors.

\section{Materials and methods}

Animal model. The experiments performed in the present study were performed in accordance with the Institutional Guidelines on the Use and Care of Laboratory Animals and approved by the Ethics Committee of Animal Experimentation of the Fourth Military Medical University (Xi'an, China). A total of 10 male spontaneously hypertensive rats (SHRs; 6 weeks old) and five male, age-matched Wistar-Kyoto (WKY) rats (6 weeks old), with body weights of 140-160 g, were obtained from the Laboratory Animal Center of the Fourth Military Medical University. All rats were housed at room temperature $23 \pm 2^{\circ} \mathrm{C}$ with free access to water and chow in a $12 \mathrm{~h}$ light/dark cycle. The SHRs were randomly divided into two groups $(n=5)$ : SHR group (SHR) and telmisartan-treated SHR group (Telmisartan). The group of WKY rats (WKY) served as a control. According to previous studies (18-21), tissue fibrosis or vascular remodeling in hypertension can be effectively decreased by treatment with telimisartan $(20 \mathrm{mg} / \mathrm{kg})$ or other drugs for 8 weeks. Therefore, the rats in the present study were administered with telmisartan $(20 \mathrm{mg} / \mathrm{kg})$ by oral gavage daily for 8 weeks.

Measurement of systolic blood pressure (SBP) and sample preparation. The rats were trained for measurement of blood pressure via a tail-cuff method for 1 week, following which SBP was measured every week using a blood pressure measurement system (Softron BP-2010A; Softron Beijing Biotechnology Co., Ltd., Beijing, China). The SBP data was collected three times under a resting state each time, and the average value was calculated. Following administration for 8 weeks, the rats were anesthetized by chloral hydrate $(10 \%$; $3 \mathrm{ml} / \mathrm{kg}$ ), blood samples were obtained, and the thoracic aortas were rinsed with PBS ( $\mathrm{pH} 7.4)$, placed in an ice-bath, partially dissected and stored at $-80^{\circ} \mathrm{C}$ for further analysis. The remaining tissue was fixed in $4 \%$ paraformaldehyde $(\mathrm{pH} 7.4)$ and then embedded in paraffin.

Assessment of chronic vasoconstriction. Vascular contractility was preliminarily evaluated using vasoactive substances in serum. The vasoconstrictor, Ang II was measured using ELISA kits (Westang Biotechnology, Shanghai, China) and the vasodilator, nitric oxide (NO) was measured using Griess reagent (Beyotime Institute of Biotechnology, Haimen, China). All procedures were performed according to the manufacturer's protocols.

Vascular reactivity. Intact vascular rings were separated and mounted in organ chambers, followed by incubation with Krebs buffer (118 mM NaCl, $4.75 \mathrm{mM} \mathrm{KCl,} 25 \mathrm{mM} \mathrm{NaHCO}_{3}$, $1.2 \mathrm{mM} \mathrm{MgSO}_{4}, 2 \mathrm{mM} \mathrm{CaCl}_{2}, 1.2 \mathrm{mM} \mathrm{KH}_{2} \mathrm{PO}_{4}$ and $11 \mathrm{mM}$ glucose) aerated with $95 \% \mathrm{O}_{2}$ and $5 \% \mathrm{CO}_{2}$ at $37^{\circ} \mathrm{C}$ for $30 \mathrm{~min}$. The vascular rings were stretched to $1.5 \mathrm{~g}$ of resting tension for $60 \mathrm{~min}$. Following pre-treatment with $10^{-6} \mathrm{M}$ phenylephrine, the vascular rings were treated with a cumulative concentration of acetylcholine (Ach; $10^{-10}-10^{-5} \mathrm{M}$ ). Vascular relaxation was evaluated by the relaxant response to Ach.

Oxidative stress. Systematic and vascular oxidative stresses were assessed by measuring the levels of superoxide dismutase (SOD), glutathione peroxidase (GPx) and malondialdehyde (MDA) in blood samples and vascular walls. Blood samples and vascular homogenates were collected and centrifuged at $1,400 \mathrm{x} \mathrm{g}$ for $10 \mathrm{~min}$ at $4^{\circ} \mathrm{C}$, the supernatant was obtained and respective kits were used for measurement (Jiancheng Bioengineering Institute, Nanjing, China). All experimental procedures were performed according to the manufacturer's protocols.

Hematoxylin and eosin $(H \& E)$ staining and morphological analysis. Thoracic aortas under relaxed conditions were embedded in paraffin, cut into $4-\mu \mathrm{m}$ thick sections and mounted on glass slides. The integral cross section of the vascular wall was stained using H\&E staining and visualized, and images were captured under a light microscope for the analysis of morphological features. The vascular external (Ae) and internal areas (Ai) were measured using a computerized system (cellSens version 1.7, Olympus Corporation, Tokyo, Japan). The vascular cross-sectional area was calculated as Ae-Ai, and the average wall thickness was calculated as follows: $\left[(4 \mathrm{Ae} / \pi)^{-2}-(4 \mathrm{Ai} / \pi)^{-2}\right] / 2$. The $\mathrm{M} / \mathrm{L}$ ratio was also calculated via the media thickness/lumen diameter.

Masson staining. The vascular slices were deparaffinized and subjected to Masson staining. Images of five microscopic fields were captured (magnification, x200) under an inverted fluorescence microscope (Olympus IX71, Olympus Corporation) and analyzed using Image-Pro Plus 6.0 (Media Cybernetics, Inc., Rockville, MD, USA).

Immunofluorescence and immunohistochemistry. The vascular sections were incubated with anti-TGF- $\beta 1$ antibody $(1: 200$; Bioss, Beijing,China; cat.no.bs-0086R) for $1 \mathrm{~h}$ at $37^{\circ} \mathrm{C}$, following which anti-rabbit rhodamine-conjugated secondary antibody (1:500; CWbiotech, Beijing, China; cat. no. CW2035M) was added for $1 \mathrm{~h}$ at room temperature. Images were captured under a fluorescent microscope (magnification, x200). The red fluorescence indicated TGF- $\beta 1$ expression. Immunohistochemical staining was also performed to assess the expression of Smad3. The vascular sections were incubated with rabbit anti-Smad3 
A

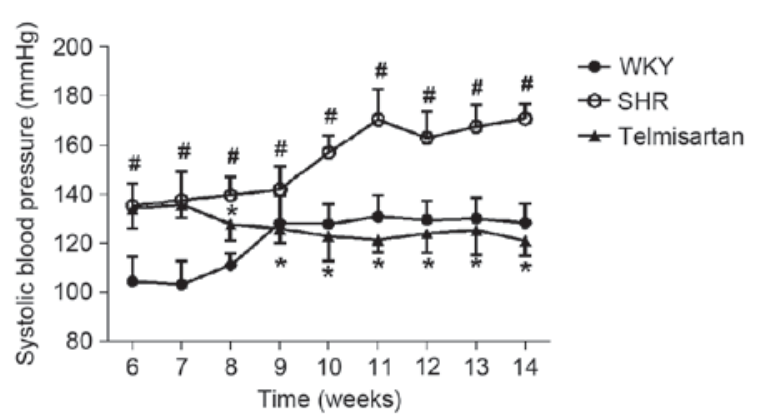

B

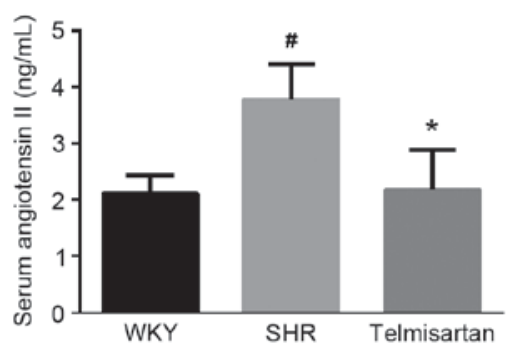

C

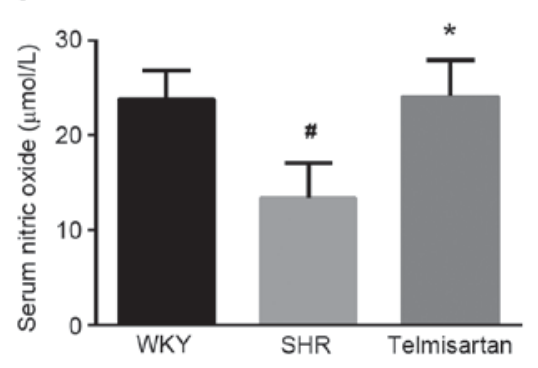

D

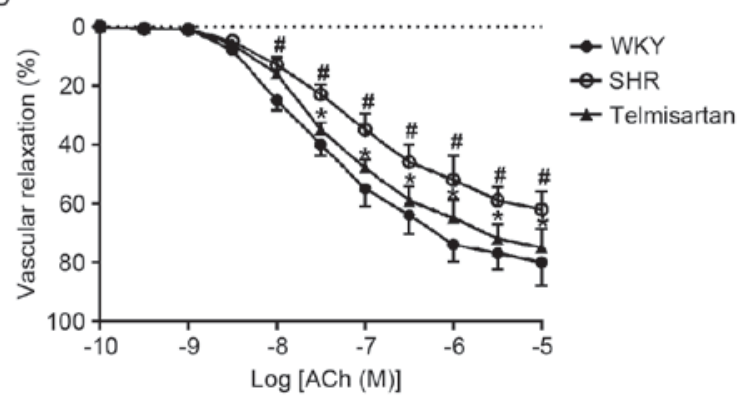

Figure 1. Telmisartan decreases SBP and restores vascular reactivity of SHR. (A) Effects on SBP. (B) Angiotensin II levels in serum. (C) Nitric oxide concentration in serum. (D) Vascular reactivity. The results show that telmisartan decreased SBP, attenuated vascular contractility by restoring angiotensin II and nitric oxide in serum, and increased vascular relaxation. Data are presented as the mean \pm standard deviation. D. ${ }^{\prime \prime} \mathrm{P}<0.05$, vs. WKY; ${ }^{*} \mathrm{P}<0.05$, vs. SHR. SBP, systolic blood pressure; WKY, Wistar-Kyoto rats; SHR, spontaneously hypertensive rats.

antibody (1:100; Abcam, Cambridge, UK; cat. no. ab40854) for $1 \mathrm{~h}$ at $37^{\circ} \mathrm{C}$. Anti-rabbit HRP-conjugated secondary antibody (CWbiotech; cat. no. CW0159S) was then added for $30 \mathrm{~min}$ at $37^{\circ} \mathrm{C}$. The expression of $\mathrm{Smad} 3$ was evaluated according to the size of the dark brown-stained region in the entire section. Images were captured (magnification, x200) with a microscope linked to a computerized imaging system (cellSens version 1.7, Olympus Corporation). The mean optical density values of immunofluorescence and immunohistochemistry were calculated using Image-Pro Plus 6.0 software.

Western blot analysis. The aortas from the rats of WKY and SHR groups were lysed with protein lysis buffer (Beyotime Institute of Biotechnology) containing 1\% PMSF (1 mM) and phosphatase inhibitor (TIANDZ 80809-1; 1\%) in an ice-bath. The lysate was then collected and centrifuged at $4^{\circ} \mathrm{C}$. The supernatant was collected and protein extracts were quantified using a BCA protein assay kit (Beyotime Institute of Biotechnology). The protein $(30 \mu \mathrm{g})$ was subjected to SDS-PAGE with a $10 \%$ polyacrylamide gel, following which proteins were transferred onto nitrocellulose membranes. The membranes were incubated with following primary antibodies overnight at $4^{\circ} \mathrm{C}$ : Anti-TGF- $\beta$ (1:1,000; Abcam, cat. no. ab64715); anti-Smad3 (1:1,000; Abcam; cat. no. ab40854); anti-p-Smad3 (1:400; Bioss; cat. no. BS-3425R); anti- $\alpha$-SMA (1:400; Boster Systems, Inc., Pleasanton, CA, USA; cat. no. BM0002) and anti-collagen 1 (1:400; Boster Systems, Inc.; cat. no. BA0325). Anti- $\beta$-actin (1:500; Sinopept Biotechnology Co., Ltd., Beijing, China; cat. no. $41302 \mathrm{M}$ ) served as a loading control. The membranes were then incubated with the following secondary antibodies for $1 \mathrm{~h}$ at room temperature: HRP-mouse monoclonal (1:1,000;
CWbiotech; cat. no. CW0102) anti-TGF- $\beta 1, \alpha-S M A$ and $\beta$-actin and HRP-rabbit monoclonal (1:1,000; CWbiotech; cat. no. CW0103) anti-Smad3, p-Smad3 and collagen1. Immunoblots were detected using Tanon-4200 and recorded using a Tanon GIS detection system (Tanon Science and Technology Co., Ltd., Shanghai, China). Signal intensities were measured using the Image J program (version 1.49, National Institutes of Health, Bethesda, MA, USA).

Statistical analysis. All data are presented as the mean \pm standard deviation and analyzed using SPSS 17.0 (SPSS, Inc., Chicago, IL, USA). Statistic variables were compared using one-way analysis of variance, followed by the LSD t-test. $\mathrm{P}<0.05$ was considered to indicate a statistically significant difference.

\section{Results}

Telmisartan decreases SBP and attenuates chronic vasoconstriction. The animals in the SHR group exhibited higher SBP at 6 weeks, which continuously increased during the subsequent 8 weeks of administration. Telmisartan significantly decreased SBP during the 8 weeks of treatment $(\mathrm{P}<0.05$; Fig. 1A). To demonstrate the pharmacological effects of telmisartan on vascular contractility, the levels of NO and Ang II in the blood samples were measured. Compared with the WKY group, the SHR group exhibited higher Ang II content, but lower NO content. Telmisartan restored their levels of expression $(\mathrm{P}<0.05$; Fig. $1 \mathrm{~B}$ and $\mathrm{C})$. These results suggested that the animals in the SHR group developed chronic vasoconstriction, which was effectively attenuated by telmisartan treatment. 
Table I. Assessment of oxidative stress in blood samples and vascular tissues.

\begin{tabular}{lccccccc}
\hline & \multicolumn{3}{c}{ Blood } & & \multicolumn{3}{c}{ Vascular tissue } \\
\cline { 2 - 3 } \cline { 7 - 8 } Group & SOD (units) & GPx (units) & MDA (nM/l) & & SOD (units) & GPx (units) & MDA (nM/l) \\
\hline WKY & $202.83 \pm 8.75$ & $800.60 \pm 49.96$ & $8.22 \pm 0.67$ & & $107.21 \pm 15.17$ & $399.06 \pm 12.97$ & $4.91 \pm 0.62$ \\
SHR & $120.90 \pm 13.25^{\mathrm{a}}$ & $636.00 \pm 34.38^{\mathrm{a}}$ & $11.52 \pm 1.34^{\mathrm{a}}$ & & $72.20 \pm 9.33^{\mathrm{a}}$ & $305.64 \pm 13.11^{\mathrm{a}}$ & $7.99 \pm 1.44^{\mathrm{a}}$ \\
Telmisartan & $195.39 \pm 22.20^{\mathrm{b}}$ & $759.40 \pm 24.27^{\mathrm{b}}$ & $7.01 \pm 0.74^{\mathrm{b}}$ & & $95.00 \pm 10.33^{\mathrm{b}}$ & $384.49 \pm 13.57^{\mathrm{b}}$ & $5.56 \pm 0.70^{\mathrm{b}}$ \\
\hline
\end{tabular}

Telmisartan decreased levels of MDA, and restored bioactivities of SOD and GPx in the blood and thoracic aortic vessel walls. Data are expressed as the mean \pm standard deviation $(\mathrm{n}=5)$. ${ }^{\mathrm{a}} \mathrm{P}<0.05$, vs. WKY group; ${ }^{\mathrm{b}} \mathrm{P}<0.05$, vs. SHR group. $\mathrm{SOD}$, superoxide dismutase; GPx, glutathione peroxidase; MDA, malondialdehyde; WKY, Wistar-Kyoto rats; SHR, spontaneously hypertensive rats.

Telmisartan restores vascular reactivity. Vascular reactivity was evaluated by the cumulative Ach-mediated vascular relaxant response. Compared with the WKY group, the vascular rings from the SHR group pre-contracted by phenyphrine $\left(10^{-6} \mathrm{M}\right)$ exhibited decreased vascular relaxation to Ach, which was enhanced by telmisartan treatment. These results indicated that telmisartan restored vascular reactivity and increased vascular relaxation (Fig. 1D).

Telmisartan decreases oxidative stress. The MDA content and the bioactivities of SOD and GPx were measured in blood samples and vascular tissues to determine whether telmisartan alleviated oxidative stress. The results showed that MDA was significantly increased, whereas the enzymatic reaction activities of SOD and GPx were notably decreased in the blood and vascular wall. As shown in Table I, telmisartan effectively restored the bioactivities of SOD and GPx, and decreased the levels of MDA in the blood and vascular tissues $(\mathrm{P}<0.05)$.

Telmisartan normalizes the thoracic aorta morphologically. The results of the H\&E staining showed that telmisartan improved vascular remodeling in SHR group (Fig. 2A). The vascular wall parameters were measured under a computer-linked microscope. The SHR group showed increased vascular cell number, cross-sectional area, vascular media thickness and $\mathrm{M} / \mathrm{L}$ ratio $(\mathrm{P}<0.05)$. Telmisartan significantly reversed these pathological alterations and restored vascular wall parameters to normal levels (Fig. 2B-E).

Telmisartan ameliorates collagen deposition. Collagen deposition is another feature of vascular remodeling, which constitutes a major component of ECM. As shown by the Masson staining (Fig. 3A), telmisartan markedly decreased collagen deposition in the aorta. Collagen deposition was semi-quantified by the mean density of the blue area, and the data showed that telmisartan significantly reduced collagen deposition $(\mathrm{P}<0.05$; Fig 3B). Similarly, the expression of collagen-1 in the vascular walls of the SHR group was significantly decreased by telmisartan treatment, as shown in the results of the western blot analysis $(\mathrm{P}<0.05$; Fig. $3 \mathrm{C}$ and $\mathrm{D})$.

Telmisartan downregulates the expression of TGF- $\beta 1$. To clarify whether TGF- $\beta 1$ was involved in the vasoprotective effect of telmisartan, the expression of TGF- $\beta 1$ was evaluated using immunofluorescence in the aorta. As shown in Fig. 4A, the SHR group exhibited a higher expression level of TGF- $\beta 1$, compared with that in the WKY group, and this was significantly decreased by telmisartan (Fig. 4B; $\mathrm{P}<0.05$ ). Consistently, telmisartan significantly decreased the protein level of TGF- $\beta 1$ in the aortas of the SHR group, as shown in the results of the western blot analysis $(\mathrm{P}<0.05$; Fig. $4 \mathrm{C}$ and $\mathrm{D})$.

Telmisartan decreases levels of p-Smad3/Smad3. Immunohistochemistry was used to assess the expression of Smad3 in the aorta. As shown in Fig. 5A, the expression of Smad3 in the vascular walls of the SHR group was increased, and was effectively decreased by telmisartan (Fig. 5B; $\mathrm{P}<0.05$ ). The results of the western blot analysis showed similar results; the protein levels of Smad3 and p-Smad3 in the aorta were significantly decreased by telmisartan treatment $(\mathrm{P}<0.05$; Fig. 5C-E).

\section{Discussion}

As an important pathological alteration in the vasculature, vascular remodeling has been increasingly regarded as a major risk of hypertension. The TGF- $\beta 1 /$ Smads pathway is critical in this process (22). However, evidence shows that Ang II and ROS induce the activation of TGF- $\beta 1(23,24)$. Ang II can induce the expression of TGF- $\beta 1$ receptors and the phosphorylation of Smads, sensitizing this fibrotic pathway $(25,26)$. Accordingly, drugs inhibiting the activity of Ang II, or in combination with ROS inhibition, may prevent the activation of TGF- $\beta 1$ and attenuate vascular remodeling. In the present study, it was found that telmisartan effectively decreased vascular remodeling in hypertension, and exerted vasoprotective effects through inhibiting the TGF- $\beta 1 / \mathrm{Smad} 3$ pathway associated with antihypertensive and antioxidant effects.

It has been found that hypertensive animals show vascular remodeling manifested with notable vascular wall changes, higher collagen deposition and impaired vascular relaxation, which are associated with increased expression and activation of TGF- $\beta 1 / \mathrm{Smad} 3$. In accordance with a previous study, the vascular remodeling process may result from chronic vasoconstriction and oxidative stress induced by Ang II (27). Telmisartan, as a targeted AT1 inhibitor, can efficiently decrease the activity of Ang II and Ang II-induced oxidative stress. In the present study, SBP was effectively decreased in the SHR group (Fig. 1A), and the condition of chronic 


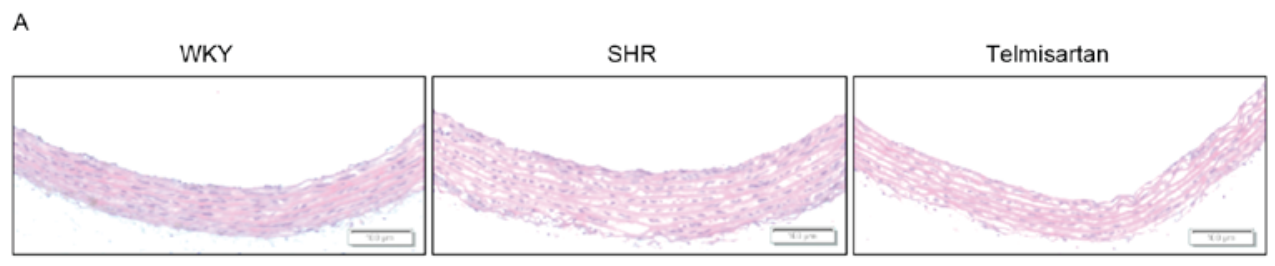

B

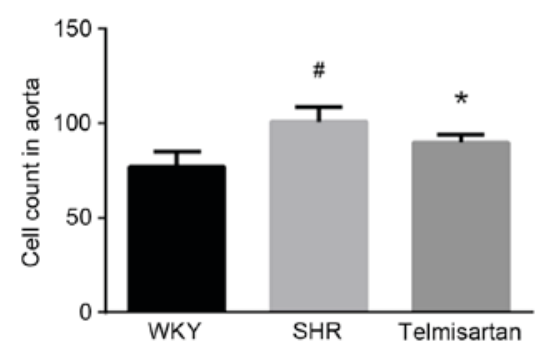

D

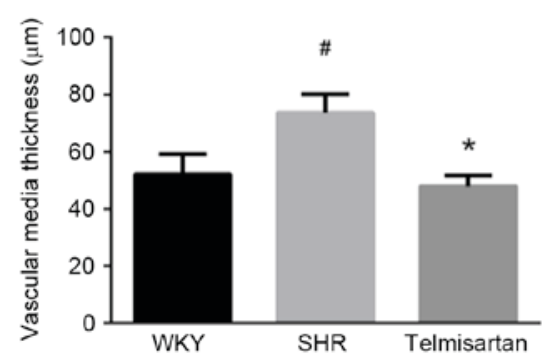

C

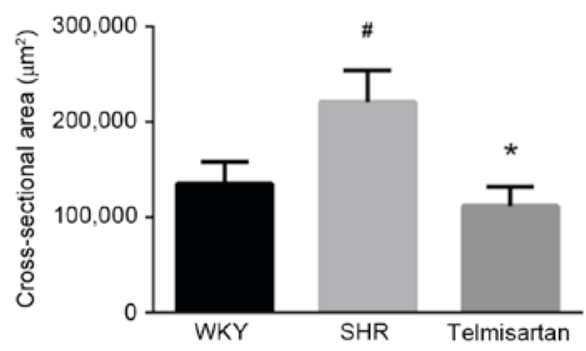

$\mathrm{E}$

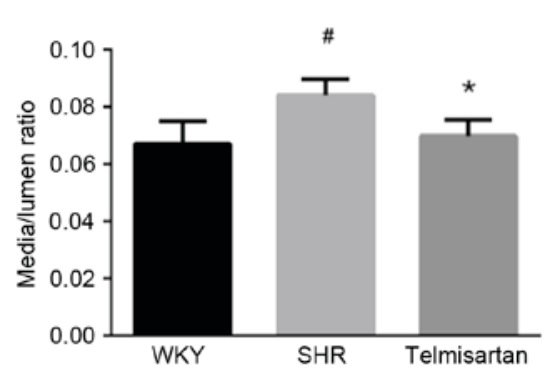

Figure 2. Telmisartan restores vascular morphological alterations. (A) Representative images of Hematoxylin and eosin staining of aortic samples (magnification, x100). (B) Relative cell count of vascular walls. (C) Vascular cross-sectional area. (D) Vascular wall thickness. (E) Media/lumen ratio. Data are presented as the mean \pm standard deviation. ${ }^{\#} \mathrm{P}<0.05$, vs. WKY; ${ }^{*} \mathrm{P}<0.05$, vs. SHR. WKY, Wistar-Kyoto rats; SHR, spontaneously hypertensive rats.

A
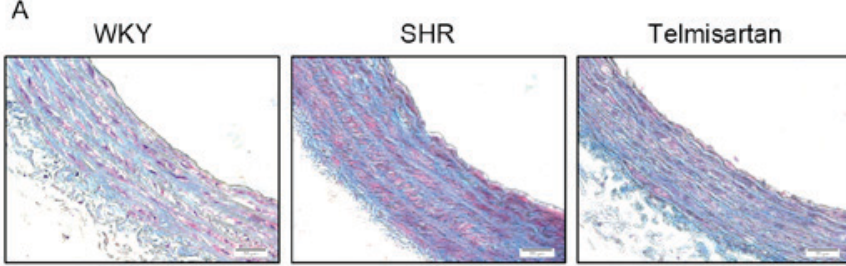

C

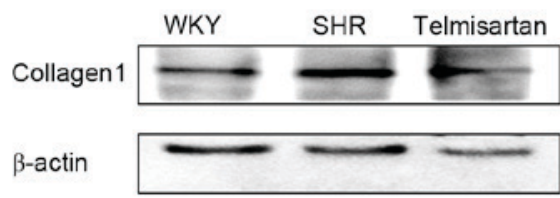

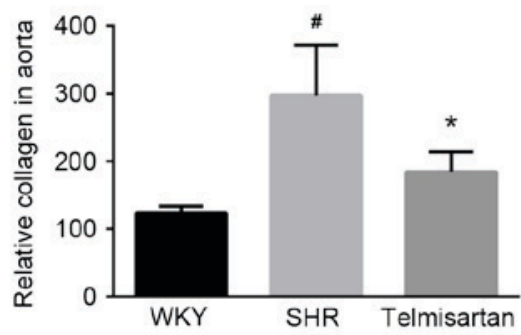

D

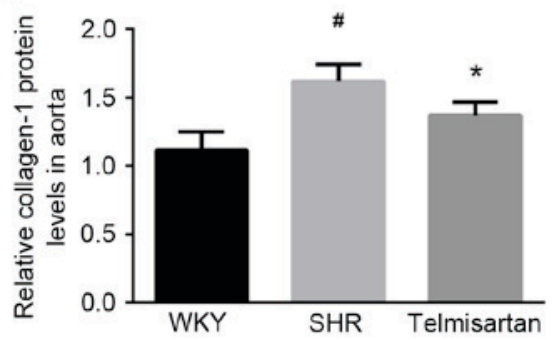

Figure 3. Telmisartan decreases collagen deposition. (A) Masson staining of aortas (magnification, x200). (B) Semi-quantitative analysis of collagen deposition. (C) Assessment of protein levels of collagen-1 in aortas using western blot analysis and (D) quantification. Data are presented as the mean \pm standard deviation. "P<0.05, vs. WKY; ${ }^{\text {P }}<0.05$, vs. SHR. WKY, Wistar-Kyoto rats; SHR, spontaneously hypertensive rats.

vasoconstriction was improved by restoring the levels of Ang II and NO in the blood (Fig. 1B and C). Of note, vascular reactivity was recovered and vascular relaxation was significantly increased by telmisartan treatment $(\mathrm{P}<0.05$; Fig. 1D).
As shown in Table I, treatment with telmisartan markedly decreased oxidative stress through restoration of the bioactivities of SOD and GPx and a decrease in the level of MDA. Taken together, these results suggested that telmisartan not 

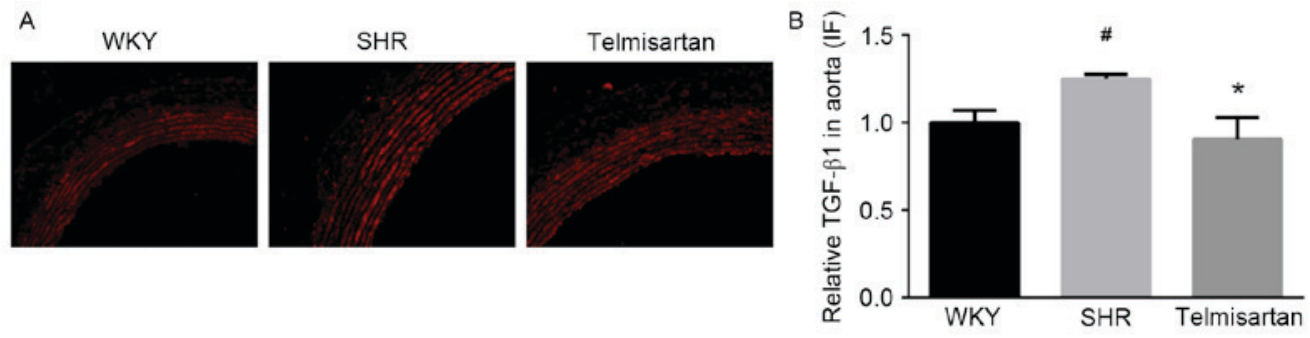

C

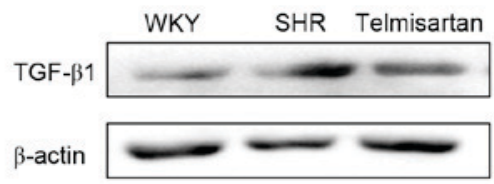

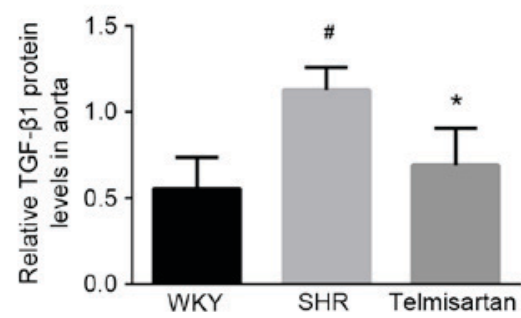

Figure 4. Telmisartan decreases the expression of TGF- $\beta 1$. (A) Expression of TGF- $\beta 1$ in the thoracic aorta was assessed using immunofluorescence (magnification, x200). (B) Semi-quantitative analysis shows that telmisartan significantly decreased the expression of TGF- $\beta 1$ in the aortas of hypertensive animals. $(C$ and D) Relative protein levels of TGF- $\beta 1$ in aortas were assessed using western blot analysis. The result showed that telmisartan decreased the expression of TGF- $\beta 1$. Data are presented as the mean \pm standard deviation. ${ }^{*} \mathrm{P}<0.05$, vs. WKY; ${ }^{*} \mathrm{P}<0.05$, vs. SHR. TGF- $\beta 1$, transforming growth factor- $\beta 1$; WKY, Wistar-Kyoto rats; SHR, spontaneously hypertensive rats.

A
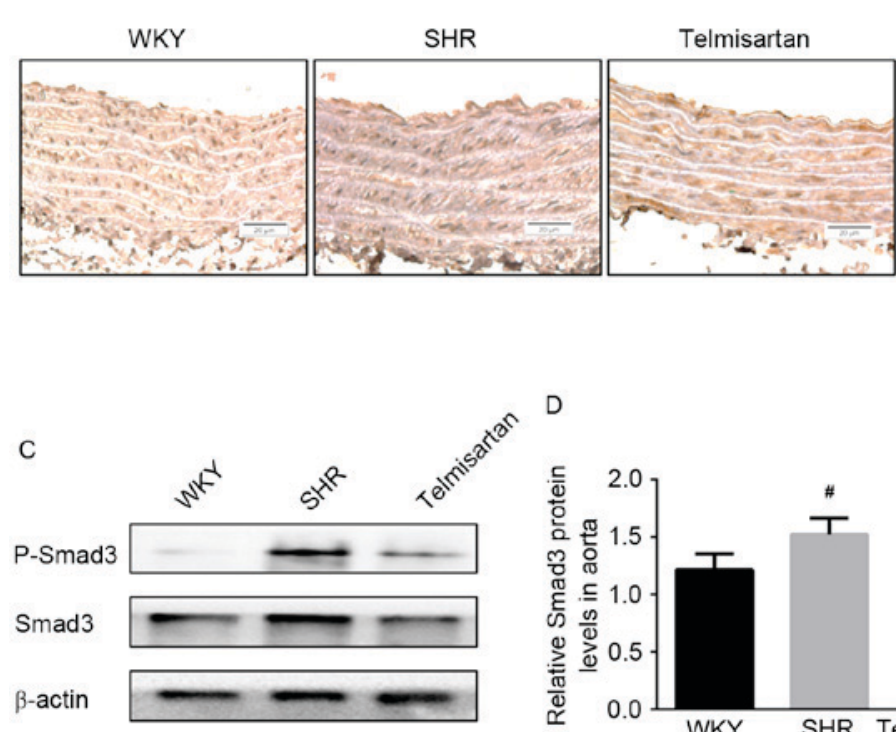

D

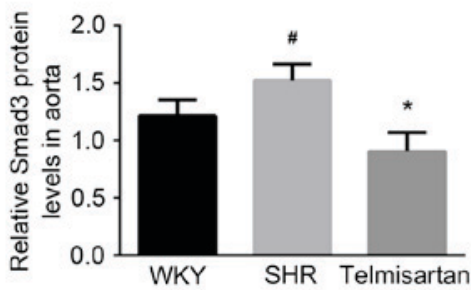

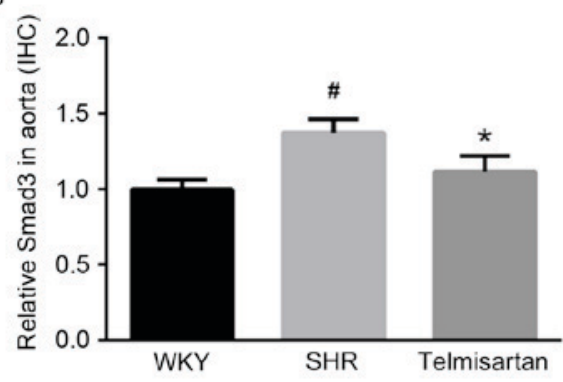

$\mathrm{E}$

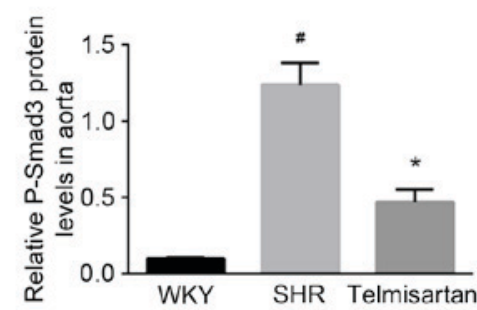

Figure 5. Telmisartan decreases the levels of Smad3 and p-Smad3. (A) Expression of Smad3 was assessed by immunohistochemistry (magnification, x200) and (B) optical density was analyzed using Image-Pro Plus 6.0. (C) Western blot analysis revealed that telmisartan effectively decreased the level of (D) Smad3, and inhibited its activation by decreasing the level of (E) $\mathrm{p}-\mathrm{Smad} 3$. Data are presented as the mean \pm standard deviation. ${ }^{*} \mathrm{P}<0.05$, vs. WKY; ${ }^{*} \mathrm{P}<0.05$, vs. SHR. Smad3, small mothers against decapentaplegic 3; p-Smad3, phosphorylated Smad3; WKY, Wistar-Kyoto rats; SHR, spontaneously hypertensive rats.

only improves hypertensive conditions, but also attenuates oxidative stress in the hypertensive animals.

The present study found that the SHR group exhibited severe vascular remodeling, compared with the WKY group, which was effectively improved by telmisartan. As shown in Fig. 2, vascular cell number, cross-sectional area, media thickness and $\mathrm{M} / \mathrm{L}$ ratio in the SHR group were significantly increased, whereas telmisartan significantly ameliorated these pathological alterations and restored vascular wall parameters to normal levels. Collagen deposition in the vascular wall is another important feature of vascular remodeling, which is predominantly regulated by the TGF- $\beta 1 / \mathrm{Smad} 3$ pathway, and affects vascular tone and structure (28). As shown using Masson staining, telmisartan markedly reduced collagen deposition in the SHR group (Fig. 3A and B). Consistently, the protein level of collagen-1 in the vascular wall of the SHR group was reduced by telmisartan treatment (Fig. 3C and D). As mentioned above, hypertension-induced vascular remodeling involves vascular morphologic alterations, ECM accumulation, collage deposition and impaired vascular reactivity.

TGF- $\beta 1$, as a typical extracellular fibrotic growth factor, regulates a set of cellular responses, including cell growth, 
differentiation, apoptosis, and the cell secretion of ECM and collagens, thus being critical in fibrotic pathology $(25,29)$. It activates receptors on membranes, phosphorylates R-Smads (Smad2/3) (30), and modulates gene expression. The modulation of ECM is reported to be predominantly Smad3-dependent (9). TGF- $\beta 1$ is vulnerable to multiple stimuli. The activation of RAS is closely associated with this pathway; for example, Ang II stimulates the activation of TGF- $\beta 1$ and expands the signal (26). ROS induced by Ang II, vasoconstriction, or higher blood pressure also activated the expression of TGF- $\beta 1$ (31) and promoted fibrotic pathologic alterations $(6,32)$. As reported previously, AT1 inhibitor notably attenuates aortic remodeling in diabetic rats with involvement of the TGF- $\beta 1 / \mathrm{Smads}$-associated profibrotic pathway (33). In addition, inhibiting Ang II-induced vascular ROS effectively prevents vascular remodeling $(34,35)$. Accordingly, drugs inhibiting AT1 or combined with antioxidant effects may have a higher capacity to decrease the expression of TGF- $\beta 1$ and prevent vascular remodeling.

Consistent with previous studies $(25,29)$, the present study showed that telmisartan significantly decreased the expression of TGF- $\beta 1$, which was higher in the SHR vascular wall, as shown by the immunofluorescence $(\mathrm{P}<0.05$; Fig. $4 \mathrm{~A}$ and $\mathrm{B})$. The protein level of TGF- $\beta 1$ in the SHR group was also decreased by telmisartan treatment (Fig. 4C and D). Similarly, as shown by the immunohistochemistry, the expression of Smad3 was inhibited by telmisartan (Fig. 5A and B). The protein levels of Smad3 and p-Smad3 were further assessed using western blot analysis, and the results showed that Smad3 and the active form, p-Samd3 were decreased by telmisartan treatment in hypertensive animals (Fig. 4C-E). Consistently (33), these results indicated that TGF- $\beta 1$, as an important fibrotic factor, was inhibited by telmisartan, and the canonical Smad3 pathway was effectively prevented by decreasing the levels of Smad3 and p-Smad3. In this way, telmisartan exerted vasoprotective effects and decreased vascular remodeling. In the present study, vascular cell differentiation was also evaluated by $\alpha$-SMA, a biomarker of myofibroblasts regulated by TGF- $\beta(11,36)$. However, no significant difference was found in $\alpha$-SMA among groups.

In the present study, telmisartan improved vascular contractility and oxidative stress, prevented profibrotic mechanisms, and exerted therapeutic effects on vascular remodeling in hypertension. These findings provide novel insights into the beneficial effect of telmisartan on vascular remodeling and the involvement of the TGF- $\beta 1 / \mathrm{Smad} 3$ pathway. Therefore, inhibition of the Ang II-dependent activation of TGF- $\beta 1 / \mathrm{Smad} 3$ in essential hypertension can be regarded as an important mechanism, by which telmisartan prevents vascular remodeling. The pathogenesis of essential hypertension and vascular remodeling is complex, and the potential mechanisms contributing to this protective effect remain to be fully elucidated. Taken together, the present study demonstrated that telmisartan effectively antagonized Ang II activity and Ang II-induced oxidative stress, inhibiting the TGF- $\beta 1 / \mathrm{Smad} 3$ pathway to protect against vascular remodeling in hypertension.

In conclusion, telmisartan effectively decreased blood pressure and chronic vasoconstriction, restored vascular reactivity and wall parameters, and decreased collagen deposition and TGF- $\beta 1 / \mathrm{Smad} 3$ activation. These results confirmed that telmisartan effectively decreased vascular remodeling, at least in part, through the TGF- $\beta 1 / \mathrm{Smad} 3$ pathway.

\section{Acknowledgements}

This study was financially supported by the National Natural Science Foundation of China (grant nos. 81373947, 81501003, 81673631 and 81603385) and the China Postdoctoral Science Foundation (grant no. 2015M580465).

\section{References}

1. Van Bortel LM: What does intima-media thickness tell us? J Hypertens 23: 37-39, 2005.

2. Intengan HD and Schiffrin EL: Vascular remodeling in hypertension roles of apoptosis, inflammation, and fibrosis. Hypertension 38: 581-588, 2001.

3. Heusch G, Libby P, Gersh B, Yellon D, Böhm M, Lopaschuk G and Opie L: Cardiovascular remodelling in coronary artery disease and heart failure. Lancet 383: 1933-1943, 2014.

4. Renna NF, de Las Heras N and Miatello RM: Pathophysiology of vascular remodeling in hypertension. Int J Hypertension 2013: Article ID 808353, 2013.

5. Montezano AC, Nguyen Dinh Cat A, Rios FJ and Touyz RM: Angiotensin II and Vascular Injury. Curr Hypertens Rep 16: 431, 2014.

6. Harrison DG, Gongora MC, Guzik TJ and Widder J: Oxidative stress and hypertension. J Am Soc Hypertens 1: 30-44, 2007.

7. Popovic N, Bridenbaugh EA, Neiger JD, Hu JJ, Vannucci M, Mo Q, Trzeciakowski J, Miller MW, Fossum TW, Humphrey JD and Wilson E: Transforming growth factor-beta signaling in hypertensive remodeling of porcine aorta. Am J Physiol Heart Circ Physiol 297: H2044-H2053, 2009.

8. Heldin C and Moustakas A: Role of smads in TGF- $\beta$ signaling. Cell Tissue Res 347: 21-36, 2012.

9. Flanders KC: Smad3 as a mediator of the fibrotic response. Int J Exp Pathol 85: 47-64, 2004.

10. Dobaczewski M, Bujak M, Li N, Gonzalez-Quesada C, Mendoza LH, Wang XF and Frangogiannis NG: Smad3 signaling critically regulates fibroblast phenotype and function in healing myocardial infarction. Circ Res 107: 418-428, 2010.

11. Jiang YL, Dai AG, Li QF and Hu RC: Transforming growth factor-beta1 induces transdifferentiation of fibroblasts into myofibroblasts in hypoxic pulmonary vascular remodeling. Acta Biochim Biophys Sin (Shanghai) 38: 29-36, 2006.

12. Lavoie P, Robitaille G, Agharaziia M, Ledbetter S, Lebel M and Lariviere R: Neutralization of transforming growth factor-beta attenuates hypertension and prevents renal injury in uremic rats. J Hypertens 23: 1895-1903, 2005.

13. Lan T, Huang $\mathrm{X}$ and Tan $\mathrm{H}$ : Vascular fibrosis in atherosclerosis. Cardiovasc Pathol 22: 401-407, 2013.

14. Border WA and Noble NA: Interactions of transforming growth factor-beta and angiotensin II in renal fibrosis. Hypertension 31: 181-188, 1998.

15. Waghe P, Sarath TS, Gupta P, Kandasamy K, Choudhury S, Kutty HS, Mishra SK and Sarkar SN: Arsenic causes aortic dysfunction and systemic hypertension in rats: Augmentation of angiotensin II signaling. Chem Biol Interact 237: 104-114, 2015.

16. Tieu BC, Lee C, Sun H, Lejeune W, Recinos A III, Ju X, Spratt H, Guo DC, Milewicz D, Tilton RG and Brasier AR: An adventitial IL-6/MCP1 amplification loop accelerates macrophage-mediated vascular inflammation leading to aortic dissection in mice. J Clin Invest 119: 3637-3651, 2009.

17. Martins-Oliveira A, Castro MM, Oliveira DM, Rizzi E, Ceron CS, Guimaraes D, Reis RI, Costa-Neto CM, Casarini DE, Ribeiro AA, et al: Contrasting effects of aliskiren versus losartan on hypertensive vascular remodeling. Int J Cardiol 167: 1199-1205, 2013

18. Muller P, Kazakov A, Semenov A, Jagoda P, Friedrich EB, Bohm $\mathrm{M}$ and Laufs U: Ramipril and telmisartan exhibit differential effects in cardiac pressure overload-induced hypertrophy without an additional benefit of the combination of both drugs. J Cardiovasc Pharmacol Ther 18: 87-93, 2012. 
19. Chang W, Cheng J and Chen Z: Telmisartan improves cardiac fibrosis in diabetes through peroxisome proliferator activated receptor $\delta$ (PPAR $\delta$ ): From bedside to bench. Cardiovasc Diabetol 15: 113, 2016.

20. Guimarães DA, Rizzi E, Ceron CS, Martins-Oliveira A, Gerlach RF, Shiva S and Tanus-Santos JE: Atorvastatin and sildenafil decrease vascular TGF- $\beta$ levels and MMP-2 activity and ameliorate arterial remodeling in a model of renovascular hypertension. Redox Biology 6: 386-395, 2015.

21. Castro MM, Rizzi E, Rodrigues GJ, Ceron CS, Bendhack LM, Gerlach RF and Tanus-Santos JE: Antioxidant treatment reduces matrix metalloproteinase-2-induced vascular changes in renovascular hypertension. Free Radical Bio Med 46: 1298-1307, 2009.

22. Lu G, Xu S, Peng L, Huang Z, Wang Y and Gao X: Angiotensin II upregulates Kv1.5 expression through ROS-dependent transforming growth factor-beta1 and extracellular signal-regulated kinase $1 / 2$ signalings in neonatal rat atrial myocytes. Biochem Bioph Res Commun 454: 410-416, 2014.

23. Cabello-Verrugio C, Acuña MJ, Morales MG, Becerra A, Simon F and Brandan E: Fibrotic response induced by angiotensin-II requires NAD $(\mathrm{P}) \mathrm{H}$ oxidase-induced reactive oxygen species (ROS) in skeletal muscle cells. Biochem Bioph Res Commun 410: 665-670, 2011

24. Morales MG, Vazquez Y, Acuña MJ, Rivera JC, Simon F, Salas JD, Álvarez Ruf J, Brandan E and Cabello-Verrugio C: Angiotensin II-induced pro-fibrotic effects require p38MAPK activity and transforming growth factor beta 1 expression in skeletal muscle cells. Int J Biochem Cell Biol 44: 1993-2002, 2012.

25. Yang F, Chung AC, Huang XR and Lan HY: Angiotensin II induces connective tissue growth factor and collagen I expression via transforming growth factor-beta-dependent and -independent smad pathways: The role of Smad3. Hypertension 54: 877-884, 2009.

26. Siegert A, Ritz E, Orth S and Wagner J: Differential regulation of transforming growth factor receptors by angiotensin II and transforming growth factor-betal in vascular smooth muscle. J Mol Med (Berl) 77: 437-445, 1999.

27. Fang H, Chen W, Gao Y, Shen Y and Luo M: Molecular mechanisms associated with angiotensin-converting enzyme-inhibitory peptide activity on vascular extracellular matrix remodeling. Cardiology 127: 247-255, 2014.
28. Xu L, Li L, Zhao P, Qi L, Li P, Gao J and Fei G: Total polysaccharide of Yupingfeng protects against bleomycin-induced pulmonary fibrosis via inhibiting transforming growth factor- $\beta$ 1-mediated type I collagen abnormal deposition in rats. J Pharm Pharmacol 12: 1786-1795, 2014.

29. Gao F, Chambon P, Tellides G, Kong W, Zhang X and Li W: Disruption of TGF- $\beta$ signaling in smooth muscle cell prevents flow-induced vascular remodeling. Biochem Biophys Res Commun 454: 245-250, 2014.

30. Zhang Y, Elsik M, Edgley AJ, Cox AJ, Kompa AR, Wang B, Tan CY, Khong FL, Stapleton DI, Zammit S, et al: A new anti-fibrotic drug attenuates cardiac remodeling and systolic dysfunction following experimental myocardial infarction. Int J Cardiol 168: 1174-1185, 2013.

31. Iglesias-De L MC, Ruiz-Torres P, Alcamí J, Díez-Marqués L, Ortega-Velázquez R, Chen S, Rodríguez-Puyol M, Ziyadeh FN and Rodríguez-Puyol D: Hydrogen peroxide increases extracellular matrix mRNA through TGF-beta in human mesangial cells. Kidney Int 59: 87-95, 2001.

32. Martinez-Lemus LA, Zhao G, Galiñanes EL and Boone M: Inward remodeling of resistance arteries requires reactive oxygen species-dependent activation of matrix metalloproteinases. Am J Physiol Heart Circ Physiol 300: H2005-H2015, 2011

33. Sun H, Zhao Y, Bi X, Li S, Su G, Miao Y, Ma X, Zhang Y, Zhang W and Zhong M: Valsartan blocks thrombospondin/transforming growth factor/Smads to inhibit aortic remodeling in diabetic rats. Diagn Pathol 10: 18, 2015.

34. Nakashima T, Umemoto S, Yoshimura K, Matsuda S, Itoh S, Murata T, Fukai T and Matsuzaki M: TLR4 is a critical regulator of angiotensin II-induced vascular remodeling: The roles of extracellular SOD and NADPH oxidase. Hypertens Res 38: 649-655, 2015.

35. CF L, Zhang J, Shen K, Gao P, Wang H, Jin X, Meng C and Fang N: Adventitial gene transfer of catalase attenuates angiotensin II-induced vascular remodeling. Mol Med Rep 11: 2608-2614, 2015.

36. Serini G and Gabbiana G: Modulation of alpha-smooth muscle actin expression in fibroblasts by transforming growth factor-beta isoforms: An in vivo and in vitro study. Wound Repair Regen 4: 278-287, 1996 